\title{
Probing the composition of sub-millisecond rotating compact stars by r-modes instability
}

\section{Giuseppe Pagliara*}

Institut für Theoretische Physik, Goethe Universität, Max-von-Laue-Str. 1, 60438 Frankfurt am Main, Germany

E-mail: pagliara@th.physik.uni-frankfurt.de

\section{Alessandro Drago}

Dipartimento di Fisica, Universitá di Ferrara and INFN Ferrara, via Saragat 1, 44100 Ferrara, Italy

E-mail: drago@fe.infn.it

\begin{abstract}
We investigate the implications of the r-modes instability on the composition of a compact star rotating at a sub-millisecond period. In particular, the only viable astrophysical scenario for such an object, wich might present inside the Low Mass X-ray Binary associated with the X-ray transient XTE J1739-285, is that it has a strangeness content. Since previous analysis indicate that hyperonic stars or stars containing a kaon condensate are unlikely because of the mass-shedding constraint, the only remaining possibility is that such an object is either a strange quark star or a hybrid quark-hadron star.
\end{abstract}

8th Conference Quark Confinement and the Hadron Spectrum September 1-6 2008

Mainz, Germany

${ }^{*}$ Speaker. 
It has been widely discussed in the literature the possibility that r-mode instabilities can very efficiently drag angular momentum from a rotating compact star if its temporal evolution in the $\Omega-$ $T$ plane (angular velocity and temperature) enters the r-mode instability window, see e.g. [1] and [2]. The size and position of that window is strictly related to the composition of the star, since it is strongly dependent on the value of bulk and shear viscosity of star matter. It is important to recall that for stars containing strangeness, as hyperon stars [3], hybrid stars [4] and strange quark stars [5] there is also a contribution to the bulk viscosity associated with the formation of strangeness. Due to this, the instability window splits into two parts: one which starts at temperatures larger than $(7 \pm 3) 10^{9} \mathrm{~K}$ (High Temperature Instability Window HTIW) and a lower temperature window at temperatures smaller than $(5 \pm 4) 10^{8} \mathrm{~K}$ (Low Temperature Instability Window, LTIW).

In Ref. [6] it is reported the evidence of a X-ray transient with a pulsed component of the emission having a frequency $f=1122 \pm 0.3 \mathrm{~Hz}$. This signal is interpreted as due to the rotation of the central neutron star. As such this object would be the most rapidly rotating compact star discovered up to now. This single observation clearly needs to be confirmed, maybe by the analysis of future $\mathrm{X}$-ray transients of the same object. The implications of this rapid rotation on the Equation of State (EOS) and in particular on the allowed values of the mass and the radius have been discussed in $[7,8]$. Here we discuss the stability with respect to r-modes of such a rapidly rotating object and in which region of the $\Omega-T$ plane the compact star is most likely located, due to its composition (for details see [9]). Let us start by discussing the simplest possibility, i.e. that the object is a neutron star. A neutron star can rapidly rotate in two cases, either if its temperature is very large, above a few MeV, or if it is recycling, spinning up due to mass accretion (see Fig. 1). Concerning the first possibility, a hot neutron star would be a newly born one, since the time needed to cool below one $\mathrm{MeV}$ is of the order of one minute. This is clearly not the case of the stellar object under discussion. Concerning recycling, it should take place on the left side of the instability window, located at lower temperatures (see Fig. 1). An important result of the analysis of [11-13] is that a neutron star can never spin-up to a rotational period shorter than $\sim 1.5 \mathrm{~ms}$. This result is based on the estimates of the temperature and of the mass accretion rates of Low Mass X-ray Binaries (LMXBs) $[14,15]$ indicating that temperatures lower than $\sim 10^{8} \mathrm{~K}$ cannot be reached. Therefore a sub-millisecond neutron star cannot be present at the center of LMXB. This conclusion is confirmed by more recent analysis, taking into account the composition stratification of the rigid crust [16] and the nonlinear development of the r-mode instability [17].

Let us now discuss the case in which the compact star contains strangeness. It has been shown, in the case of hyperon stars [18, 19] hybrid stars [20] and strange quark stars [5], that due to the large value of the bulk viscosity associated with the non-leptonic weak decays, there are two windows of instability, the LTIW and the HTIW introduced above. The HTIW does not affect significantly the angular velocity of the star because the cooling of a newly born star is so fast that there is not enough time for the r-mode instability to drag a significant fraction of the angular momentum. Therefore, the star exits the HTIW with an angular velocity close to the initial one. When the temperature drops down to a few $10^{8} \mathrm{~K}$ the star reaches the LTIW and it starts to lose angular momentum due to r-mode instability. In Fig. 1 we show examples of the instability windows in the case of a pure quark star and of a hybrid star. Notice that the position of the LTIW depends rather strongly on the mass of the strange quark $m_{s}$. For large values of $m_{s}$ the instability windows shrink considerably. In our analysis we have considered two possibilities concerning the value of $m_{s}$ : a 

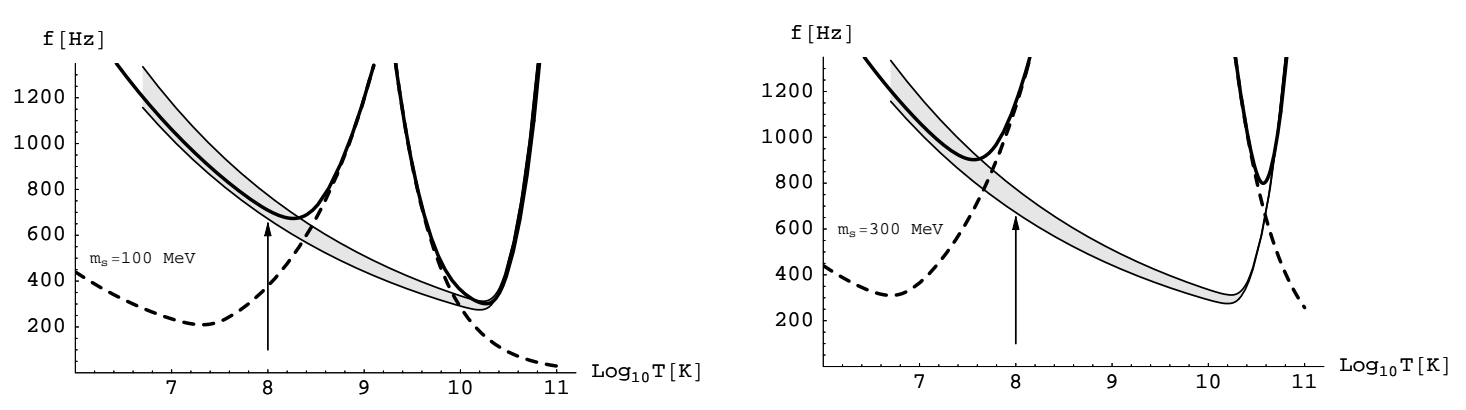

Figure 1: r-mode instability windows. The star is unstable and loses angular momentum by emitting gravitational waves in the regions above the instability lines displayed in figure. Thin solid lines correspond to neutron stars, for two extreme values of masses and radii allowed by the analysis done by [8]. The shaded area in between is representative of intermediate values of masses and radii. Dashed lines delimit the LTIW and the HTIW for strange quark stars. Thick solid lines correspond to hybrid stars. In both cases the mass and radius of the star are $M=2 M_{\odot}$ and $R=13 \mathrm{~km}$. In the left panel $m_{s}=100 \mathrm{MeV}$ and in the right panel $m_{s}=300 \mathrm{MeV}$. The bulk and shear viscosities are in general from [10]. Bulk viscosity of strange quark matter at high temperatures is from [5]. The arrow indicates the minimal temperature possible in LMXBs.

small value $\sim 100 \mathrm{MeV}$, of the order of the strange quark current mass, and a large value $\sim 300$ $\mathrm{MeV}$, similar to what has been obtained in NJL-like models.

Concerning the left side of the LTIW, it is easy to see that it cannot be used to accommodate a sub-millisecond pulsar. Indeed, in the case of hybrid or hyperonic stars the left side of the LTIW is similar to the one of neutron stars, discussed above, and we can apply here the same analysis done for a neutron star. In the case of quark stars the absence of viscous boundary layers implies that they can rotate rapidly only on the right side of the LTIW. In conclusion, stars containing strangeness can rotate at sub-millisecond periods only if they are to the right of the LTIW.

Let us now discuss the results of our analysis also considering the constraints posed onto the EOS by the mass shedding limit. The main result of [7] and of [8] is that soft EOSs, and in particular the ones based on hyperonic matter or on matter in which kaon condensation takes place, are rather unlikely. In fact, not only the range of allowed masses is rather small, but moreover the only configurations satisfying the stability constraint for mass shedding turn out to be supra massive [8], making it very difficult to use these stellar structures in a mass accretion scenario. This result confirms previous discussions of other astrophysical objects ruling out soft EOSs [21]. On the other hand, stars containing deconfined quark matter are not excluded if the quark EOS is stiff enough [22]. We conclude that the object under discussion is either a quark or a hybrid star.

We can now study the temporal evolutions of the angular velocity of the star $\Omega$, of its temperature $T$ and of the amplitude of the r-modes $\alpha$ by solving the set of differential equations given in [23] (Eqs. 15-23-24). The only technical difference in our calculation is the inclusion of the reheating associated with the dissipation of r-modes by bulk viscosity, as discussed in various papers [18-20]. Due to the reheating, the trajectory in the $\Omega-T$ plane describing the time evolution of the star follows essentially the border of the LTIW and the star keeps rotating as a sub-millisecond pulsar for a very long time, strongly dependent on the value of $m_{s}$ and ranging from $\sim 10^{2}$ years when $m_{s}=100 \mathrm{MeV}$ to $\sim 10^{8}$ years when $m_{s}=300 \mathrm{MeV}$, as shown in Fig. 2 . 


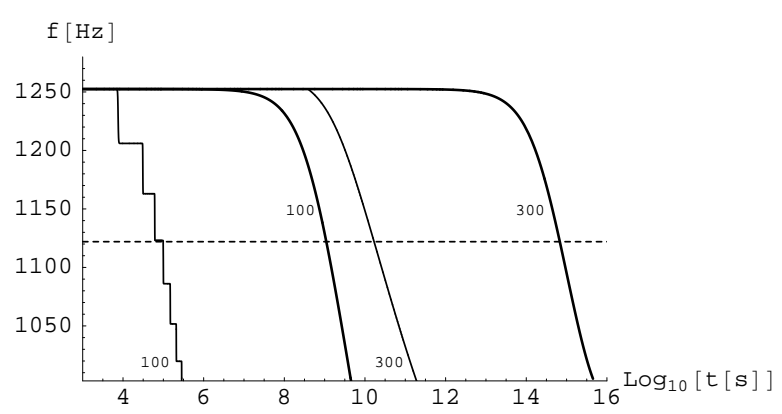

Figure 2: Time dependence of the rotational frequency of the compact star. The thin lines take into account only the reheating due to shear viscosity, the thick lines take into account also the effect of reheating due to bulk viscosity. We show results for two different values of the strange quark mass, $m_{s}=100 \mathrm{MeV}$ and $m_{s}=300 \mathrm{MeV}$. The dashed horizontal line corresponds to a frequency of $1122 \mathrm{~Hz}$.

Before discussing the possible astrophysical scenarios for a submillisecond pulsar inside a LMXB, it is important to remark two constraints that a realistic model should fulfill. First, a LMBX is an old object, with a typical age of $10^{8}-10^{9}$ years. Therefore we need to provide a mechanism allowing the central object inside the LMXB to rotate rapidly while being so old. Second, submillisecond pulsars are rather rare and there is no evidence of a uniform distribution of pulsar rotational frequencies extending from a few hundred $\mathrm{Hz}$ up to more than a $\mathrm{kHz}$. Therefore a realistic model should also indicate why most of the compact stars have not sub-millisecond periods. These two constraints can be satisfied in two different astrophysical scenarios: a first scenario in which an old hybrid or quark star is accelerated up to frequencies exceeding one $\mathrm{kHz}$ by mass accretion and a second scenario in which the quark or hybrid star is born with a submillisecond period and it is now spinning down by r-mode instability. The possible realization of these scenarios depends on two main ingredients: the value of $m_{s}$, which regulates the magnitude and position of the LTIW, and the cooling rate which determines the inner temperature $T$ of an accreting star [24]. Concerning the cooling rate, if strange quark matter is present in a compact star, direct URCA processes are possible and therefore the cooling is (generally) fast. It turns out from [24] that in this case the inner temperature is $\sim 5 \times 10^{7} \mathrm{~K}$ for a mass accretion rate of $\dot{M}=10^{-10} M_{\odot} /$ year and that the temperature scales as $T \propto \dot{M}^{1 / 6}$. Another possibility recently proposed is that, due to the formation of diquark condensates, URCA processes are strongly suppressed and the cooling turns out to be slow but, at the same time, bulk viscosity is still large enough to suppress r-mode instability [25]. In the following we discuss both fast and slow cooling.

Let us consider first the case in which $m_{s}=300 \mathrm{MeV}$. The first scenario, i.e. the star span up by accretion, can indeed be realized if $T \geq 10^{8} \mathrm{~K}$ (see right panel of Fig. 1). Such a temperature can be reached via reheating due to mass accretion. In the case of fast cooling a rather large value of mass accretion rate is needed, $\dot{M} \sim 10^{-8} M_{\odot} /$ year, and this stringent request can explain why submillisecond pulsars are rare. A model in which the cooling is slow is instead excluded, because it would be extremely easy to re-accelerate the star to very large frequencies and therefore submillisecond pulsars would not be rare. Also the second scenario in which the star is spinning down due to r-mode instabilities is possible. As shown in Fig. 2, the time spent by the star above $1122 \mathrm{~Hz}$ is of order of $10^{8}$ years and it is therefore compatible with the typical age of LMXBs. In this case submillisecond pulsars are rare because only a (small) fraction of newly born compact 
stars can rotate with submillisecond periods (see e.g. the discussions in [10]).

We consider now the case in which $m_{s}=100 \mathrm{MeV}$. The first scenario in which the star is spinning up by accretion can be realized but $T$ must be $\gtrsim 5 \times 10^{8} \mathrm{~K}$ for the star to be accelerated up to $1122 \mathrm{~Hz}$ (see left panel of Fig. 1). Such a temperature can be reached only in presence of a strong reheating due to a large mass accretion rate and slow cooling. The second scenario, i.e. the star is spinning down by r-mode instability, is ruled out since, as shown in Fig. 2, the time spent by the star above $1122 \mathrm{~Hz}$ is only of the order of 100 years.

The main uncertainties in our analysis are due to the possible existence of other damping mechanisms, taking place on the left side of the LTIW. For instance magnetic fields can be important to suppress r-mode instabilities [26], but their effect is probably negligible for frequencies exceeding $\sim 0.35 \Omega_{K}$, if the internal magnetic field is not larger than $\sim 10^{16} \mathrm{G}$. Obviously, even larger uncertainties exist concerning quark matter. As discussed above, the bulk viscosity of quark matter strongly depends on the strange quark mass and on the possible formation of a diquark condensate [27-29]. Clearly, our analysis can provide much needed constraints on the EOS of quark matter. Finally, let us stress that the outcome of our analysis is that a compact star rotating at a submillisecond period inside a LMXB can only be a quark or a hybrid star. Future observations will be important to clarify if the object at the center of XTE J1739-285 constitutes indeed an example.

\section{Acknowledgments}

G.P. thanks Sandra Sichler for many fruitful discussions.

\section{References}

[1] N. Andersson, A New class of unstable modes of rotating relativistic stars, Astrophys.J. 502 (1998) 708 [gr-qc/9706075].

[2] J. L. Friedman and S. M. Morsink, Axial Instability of Rotating Relativistic Stars, Astrophys.J. 502 (1998) 714 [gr-qc/9706073].

[3] L. Lindblom and B. J. Owen Effect of hyperon bulk viscosity on neutron star r modes, Phys. Rev.D 65 (2002) 063006 [astro-ph/0110558].

[4] A. Drago, A. Lavagno and G. Pagliara, Bulk viscosity in hybrid stars, Phys. Rev.D 71 (2005) 103004 [astro-ph/0312009].

[5] J. Madsen, Probing Strange Stars and Color Superconductivity by r-Mode Instabilities in Millisecond Pulsars, Phys. Rev. Lett. 85 (2000) 10 [astro-ph/9912418].

[6] P. Kaaret et al., Evidence of $1122 \mathrm{~Hz}$ X-Ray Burst Oscillations from the Neutron Star X-Ray Transient XTE J1739-285, Astrophys.J. 657 (2007) L97 [astro-ph / 0611716 ].

[7] G. Lavagetto et al., Constraints to the EOS of ultradense matter with model-independent astrophysical observations, (2006) [astro-ph/0612061].

[8] M. Bejger, P. Haensel and J. L. Zdunik, Rotation at $1122 \mathrm{~Hz}$ and the neutron star structure, Astron.Astrophys. 464 (2007) L49 [astro-ph/ 0612216 ].

[9] A. Drago, G. Pagliara and I. Parenti, A Compact Star Rotating at $1122 \mathrm{~Hz}$ and the r-Mode Instability, Astrophys.J. 678 (2008) L117 [0 704 . 1510]. 
[10] N. Andersson and K. D. Kokkotas, The R mode instability in rotating neutron stars, Int. J. Mod. Phys.D 10 (2001) 381 [gr-qc/ 0010102 ].

[11] L. Bildsten and G. Ushomirsky, Viscous Boundary-Layer Damping of R-Modes in Neutron Stars, Astrophys.J 529 (2000) L33 [astro-ph/9911155].

[12] Y. Levin, Runaway Heating by R-Modes of Neutron Stars in Low-Mass X-Ray Binaries, Astrophys.J. 517 (1999) L328 [astro-ph/9810471].

[13] N. Andersson, D. I. Jones, K. D. Kokkotas and N. Stergioulas, R mode runaway and rapidly rotating neutron stars, Astrophys.J. 534 (2000) L75 [astro-ph / 0002114 ].

[14] E. F. Brown and L. Bildsten, The Ocean and Crust of a Rapidly Accreting Neutron Star: Implications for Magnetic Field Evolution and Thermonuclear Flashes, Astrophys.J. 496 (1998) 915 [astro-ph/9710261].

[15] S. Bhattacharyya, A study of accretion discs around rapidly rotating neutron stars in general relativity and its applications to four low mass X-ray binaries, Astron.Astrophys. 383 (2002) 524 [astro-ph/0112178].

[16] K. Glampedakis and N. Andersson, Ekman layer damping of r-modes revisited, Mon.Not.Roy.Astron.Soc. 371 (2006) 1311 [astro-ph/ 0607105 ].

[17] R. Bondarescu, S. A. Teukolsky and I. Wasserman, Spin Evolution of Accreting Neutron Stars: Nonlinear Development of the R-mode Instability, Phys.Rev.D 76 (2007) 064019 [0704 0799 ].

[18] R. V. Wagoner, Conditions for Steady Gravitational Radiation from Accreting Neutron Stars, Astrophys.J. 578 (2002) L63 [astro-ph/ 0207589 ].

[19] A. Reisenegger and A. A. Bonacic, Millisecond Pulsars with r-Modes as Steady Gravitational Radiators, Phys. Rev. Lett. 91 (2003) 201103 [astro-ph/0303375].

[20] A. Drago, G. Pagliara and Z. Berezhiani, Gravitational wave bursts induced by r-mode spin-down of hybrid stars, Astron.Astrophys. 445 (2006) 1053 [gr-qc/ 0405145 ].

[21] F. Ozel, Soft equations of state for neutron-star matter ruled out by EXO 0748 - 676, Nature 441 (2006) 1115.

[22] M. Alford et al., Quark matter in compact stars ?, Nature 445 (2007) E7 [a st ro-ph / 0606524 ].

[23] N. Andersson, D. I. Jones and K. D. Kokkotas, Strange stars as persistent sources of gravitational waves, Mon.Not.Roy.Astron.Soc. 337 (2002) 1224 [astro-ph/0111582].

[24] J. Miralda-Escudé, P. Haensel and B. Paczynski, Thermal structure of accreting neutron stars and strange stars, Astrophys.J. 362 (1990) 572.

[25] D. Blaschke and J. Berdermann, Neutrino emissivity and bulk viscosity of iso-CSL quark matter in neutron stars, AIP Conf. Proc. 964 (2007) 290 [0710.5243].

[26] L. Rezzolla, F. K. Lamb and S. L. Shapiro, R-Mode Oscillations in Rotating Magnetic Neutron Stars, Astrophys.J. 531 (2000) L139 [astro-ph/9911188].

[27] M. Alford and A. Schmitt, Bulk viscosity in 2SC quark matter, J.Phys.G 34 (2007) 67 [nucl-th/0608019].

[28] P. Jaikumar, G. Rupak and A. W. Steiner, Viscous damping of r-mode oscillations in compact stars with quark matter, (2008) [0806.1005].

[29] M. Mannarelli, C. Manuel and B. Sa'd, Mutual friction in a cold color flavor locked superfluid and r-mode instabilities in compact stars, (2008) [0807.3264]. 